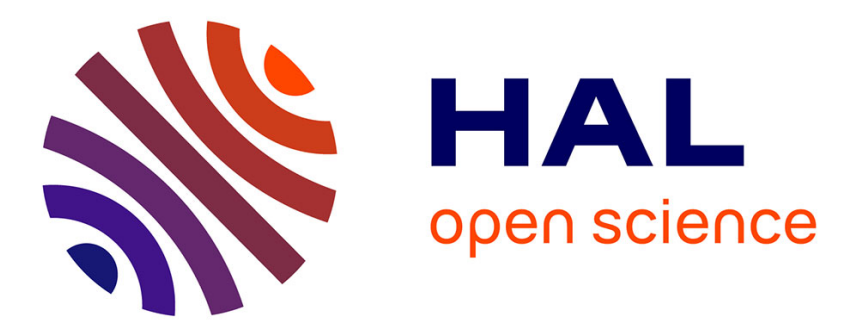

\title{
Outpatient robot-assisted radical prostatectomy: A feasibility study
}

Doria Congnard, Sébastien Vincendeau, Ahmed Lahjaouzi, Anne-Cecile Neau, Cecile Chaize, Jean-Pierre Estebe, Romain Mathieu, Hélène Beloeil

\section{- To cite this version:}

Doria Congnard, Sébastien Vincendeau, Ahmed Lahjaouzi, Anne-Cecile Neau, Cecile Chaize, et al.. Outpatient robot-assisted radical prostatectomy: A feasibility study. Urology, 2019, 128, pp.16-22. 10.1016/j.urology.2019.01.050 . hal-02086793

\section{HAL Id: hal-02086793 https://hal-univ-rennes1.archives-ouvertes.fr/hal-02086793}

Submitted on 17 Apr 2019

HAL is a multi-disciplinary open access archive for the deposit and dissemination of scientific research documents, whether they are published or not. The documents may come from teaching and research institutions in France or abroad, or from public or private research centers.
L'archive ouverte pluridisciplinaire HAL, est destinée au dépôt et à la diffusion de documents scientifiques de niveau recherche, publiés ou non, émanant des établissements d'enseignement et de recherche français ou étrangers, des laboratoires publics ou privés. 


\section{Outpatient robot-assisted radical prostatectomy: A feasibility study}

Doria Congnard ${ }^{1}$, Sébastien Vincendeau ${ }^{2}$, Ahmed Lahjaouzi ${ }^{3}$, Anne-Cecile Neau ${ }^{3}$, Cecile

Chaize $^{3}$, Jean-Pierre Estèbe ${ }^{3}$, Romain Mathieu ${ }^{4}$, Hélène Beloeil ${ }^{1}$

1 Univ Rennes, INSERM, INRA, CHU Rennes, CIC 1414, Numecan, Pole Anesthésie et Reanimation, F-35033 Rennes, France

2 Univ Rennes, CHU Rennes, CIC 1414, Department of Urology, Rennes University

Hospital, Rennes, France

3 CHU Rennes, Pole Anesthésie et Reanimation, F-35033 Rennes, France

4 Univ Rennes, CHU Rennes, Department of Urology, Rennes University Hospital, Rennes, France

Address of correspondence to Prof, Hélène Beloeil, Pôle d'Anesthésie Réanimation Chirurgicale, CHU Rennes, 2 Rue Henri Le Guilloux, 35033 Rennes Cedex 9, France.

Phone: + 33299282422

Fax: + 33299282421

E-mail: helene.beloeil@chu-rennes.fr

Conflicts of interest: none

Key words: Outpatient, robot-assisted radical prostatectomy, PADSS score

Summary ( $<\mathbf{1 2 5}$ words): Routine discharge at day 1 is possible after RARP. Improving the management of PONV may even allow outpatient management.

\section{Acknowledgments}


The authors thanks Chloé Rousseau for the statistics.

\section{Ethical statements}

The authors declare no conflict of interest

This monocentric study was performed between December 2015 and October 2017, after approval of the IRB (00010254 - 2016 - 061). According to the French law, the ethics committee (Comité d'éthique de la Société Française d'Anesthésie-réanimation (CERAR)) waived written informed consent as it was a non-interventional study.

The results were presented as an abstract during the SFAR meeting in Septembre 2018 in Paris, France

\section{Authors' Contribution statement}

Doria Congnard helped conducting the study, analysing the data, and writing the manuscript Sébastien Vincendeau helped conducting the study and editing the manuscript

Ahmed Lahjaouzi, Anne-Cecile Neau, Cecile Chaize, Jean-Pierre Estèbe helped conducting the study. They approved the final manuscript.

Romain Mathieu helped conducting the study and editing the manuscript

Hélène Beloeil helped developing the project, conducting the study, analysing the data, and writing/editing the manuscript. 


\section{Abstract}

Objectives: To evaluate the feasibility of routine outpatient management after roboticassisted radical prostatectomy (RARP). Prostate cancer is indeed the second most common cancer in men. Surgical technics have evolved from open surgery to robot-assisted surgery with a reduction of postoperative complications. Such technical improvements associated with modern anesthesia allow outpatient surgery in various types of procedures.

Materiel and Methods: After approval of the IRB, this observational prospective and monocentric study was performed in the urology unit at Rennes university hospital between December 2015 and October 2017. All patients scheduled for RARP performed by one experienced surgeon were consecutively included. The possibility of discharge was evaluated using the PADSS score until patients had a score of 9 or higher allowing their discharge. Risk factors of delayed discharge were secondarily assessed

Results: Ninety-seven patients scheduled for RARP performed by one experienced surgeon were consecutively included. Only 1 patient had a PADSS score $\geq 9$ the day of the surgery (day 0 ). $74 \%$ of the patients achieved discharge criteria one day after surgery whereas, $33 \%$ and $66 \%$ of the population was effectively discharged on day 2 and day 3, respectively. Patients with a PADSS score $\geq 9$ at day one experienced significantly less postoperative nausea and vomiting than patients with a PADSS score $\geq 9$ at day 2 or $3(7 \%$ vs $28 \%, \mathrm{p}=$ $0.01)$.

Conclusion: Outpatient RARP was not feasible in most patients. However, routine discharge at day 1 seems conceivable. Improving the management of PONV may even allow outpatient management. This progress remains to be confirmed by further studies. 


\section{Introduction}

Prostate cancer $(\mathrm{PCa})$ is the second most common cancer in men behind lung cancer with 1,095,000 new cases in worldwide in $2012^{1}$. PCa incidence varies widely across the world with the highest rates observed in Australia/New Zealand, Northern America, Western and Northern Europe with a rate greater than 80 per $100,000^{1}$. In the same countries, the mortality decreased in recent years, especially in The United States (US) with a decline of 4.3\% per year between 1996 and $2005^{2}$. This decrease is largely attributed to improvement in PCa treatments. Surgery is one of the standard treatments for non-metastatic PCa. During last decades, new surgical techniques have been proposed to replace open surgery such as laparoscopic surgery and robot-assisted surgery. These two Vatter technics have been shown to reduce respiratory and vascular complications, mortality, blood transfusion and length of hospital stay ${ }^{3}$. A decrease of surgical site infection was also reported ${ }^{4}$. Robot-assisted radical prostatectomy (RARP) has become a method of choice, with 50,000 surgeries performed in 2007 in the US ${ }^{5}$. When compared with laparoscopic surgery, the proofs of the benefit of RARP are scarce in the literature. A recent systematic review found a small improvement in postoperative pain at day 1 and up to 1 week after/surgery with laparoscopic or RARP versus open radical prostatectomy ${ }^{6}$. Likewise, a decrease of length of hospital stay was reported with RARP when compared with open radical prostatectomy ${ }^{7}$.

Improvements in surgical and anesthetic techniques allow outpatient surgery for more and more complex surgeries. It has been particularly developed in colorectal surgery ${ }^{8}$. In a study published in 2015, 5 cases of colectomy were performed in outpatient, no patients were readmitted and all patients were highly satisfied ${ }^{9}$. With 
regard to RARP, only few studies have tested the feasibility of outpatient procedure. A very small study (11 patients) published in 2010 compared a group of outpatients to a group of inpatients after RARP and showed a comparable postoperative pain control rate and overall satisfaction rate in both groups. ${ }^{10}$. Patients were highly selected in this study. To our knowledge, the feasibility of RARP as an outpatient procedure has not been shown in large numbers of patients.

Therefore, the objective of our study was to evaluate the feasibility of routine outpatient management after RARP in a large cohort of patients.

\section{Materials and methods}

This feasibility study was an observational, prospective and monocentric study. This study was performed in the urology unit at Rennes university hospital between December 2015 and October 2017, after approval of the IRB (00010254 - 2016 061). According to the French law, the ethics committee (Comité d'éthique de la Société Française d'Anesthésie-réanimation (CERAR)) waived written informed consent as it was a non-interventional study.

\section{Patients}

All RARP performed by one experienced surgeon (SV) on adult patients were consecutively included. Non-inclusion criteria were RARP performed by another surgeon. Exclusion criterion was the non-compliance with the previously defined anesthesia protocol.

\section{Anesthesia protocol}

Patients did not receive any premedication. Intraoperative protocol associated: i) induction with propofol, remifentanil using target controlled intravenous (TCl) 
anesthesia and cisatracurium, ii) adapted antibioprophylaxis as recommended (11) iii) maintenance with sevoflurane, $\mathrm{TCl}$ remifentanil and cisatracurium as needed. Analgesia and anti-emetic prophylaxis were provided with dexamethasone (8mg, single dose), ketamine (bolus $0.2 \mathrm{mg} / \mathrm{kg}$ then $0.1 \mathrm{mg} / \mathrm{kg} / \mathrm{h}$ intra operatively), lidocaine (bolus $1.5 \mathrm{mg} / \mathrm{kg}$ then $1.5 \mathrm{mg} / \mathrm{kg} / \mathrm{h}$ intra operatively). At the end of the surgery, patients without contraindication received paracetamol (1g), ketoprofen (50mg) and nefopam (20mg). Postoperative analgesia associated, morphine titration in postanesthesia care unit (PACU) and then, oral ketoprofen $(50 \mathrm{mg} / 8 \mathrm{~h})$, paracetamol $(1 \mathrm{~g} / 6 \mathrm{~h})$, and nefopam (20mg/6h). Oral oxycodone (5mg/4h) was used as rescue analgesia. Antiemetic treatment (ondansetron) was prescribed if needed. Liquid and food were allowed upon return to the urology unit. Patients were advised to chew gum. Adequate thromboprophylaxis was prescribed ${ }^{12}$.

\section{Outcome measures}

The main objective of the study was to evaluate the feasibility of outpatient management after RARP. The possibility of discharge was evaluated using the PADSS score (Post Anesthesia Discharge Scoring System), with 5 criteria (vital signs, activity, postoperative nausea and vomiting (PONV), pain, surgical bleeding) (annex 1). PADSS score was assessed one a day from D0 (day of surgery) until patients had a score of 9 or higher allowing their discharge (13)(14).

Secondary objectives were the assessment of postoperative ileus duration (defined by absence of flatus/stool and the time to first flatus/stool was recorded), quality of analgesia (evaluated by cumulative analgesics consumption), and postoperative complications. Postoperative complications were defined using the Clavien-Dindo classification ${ }^{15}$ and included surgical revision, death, unscheduled intensive care unit 
admission, surgical site infection occurring within 30 days after surgery (purulent drainage from the superficial or deep incision; organisms isolated from an aseptically obtained culture of fluid or tissue; abscess or other evidence of infection during reoperation or radiologic examination, signs or symptoms of infection (pain, redness, fever)), pulmonary infection (defined by fever, cough, oxygen requirement and lung abnormalities on the chest $\mathrm{x}$-ray) or urinary infection (defined by fever, irritative and obstructive urinary function signs (urinary frequency, retention and burning) and urine bacteria count $\geq 10^{3} \mathrm{ufc} / \mathrm{ml}$ )) renal failure (according to KDIGO criteria (16) : increase plasma creatinine $\geq 3 \mathrm{mg} / \mathrm{l}$ in 48 hours or increase in plasma creatinine $\geq 1.5$ times the baseline over the last 7 days or diuresis $<0.5 \mathrm{ml} / \mathrm{kg} / \mathrm{h}$ for 6 hours), stroke (defined by clinically evident temporary or permanent new neurologic focal deficit and confirmed by a radiologic examination) acute coronary syndrome (defined by chest pain with ST-segment modification in an electrocardiogram and elevation of troponin), acute limb ischemia (defined by pain, pallor, pulseless, perishing cold, paresthesia and paralysis). We also hypothesized that we would be able to identify risk factors for prolonged hospitalization (past Day 0).

\section{Data collection}

Aside from demographic data, we also collected the following data during the surgery: Duration of surgery, bleeding and number of red blood cells transfused, completion of lymph node dissection and/or nerve preservation, intraoperative complications (defined by any deviation from the ideal intraoperative course between skin incision and skin closure for surgical complications and between induction and recovery for anesthetic complications), and use of drains. Postoperative data were length of stay in $\mathrm{PACU}_{\overline{;}}$ discharge of PACU after 5:00 pm, cumulative opioid 
consumption in PACU, cumulative analgesics consumption during hospitalization, date of first liquid, first food, first ambulation and peripheral catheter removal, date of first flatus or stools, PONV and cumulative ondansetron consumption. Postoperative complications, PADSS score, needing of nurse interventions (different from usual monitoring defined as follows: heart rate, blood pressure, temperature, pain assessment by numerical pain scale, diuresis, aspect of the urine every 6 hours) with or with not medical call, medical intervention or deviations to standard protocol, and date of discharge (surgeon's decision) were also recorded. The date of bladder catheter's removal was not part of the data collection as it was systematically removed on the sixth postoperative day during an outpatient consultation.

\section{Statistics}

All analyses were performed with SAS $\odot$ version 9.4. and all tests were considered statistically significant at $p \leq 0.05$. Quantitative variables were described as follows: $\mathrm{n}$, mean \pm standard deviation. For qualitative variables, the size $(n)$ and percentage (\%) were presented for each category. Comparisons between groups were performed as follows: for quantitative variables, patients were compared by a Student's test when the distribution follows a normal distribution or by non-parametric tests of Mann-Whitney Wilcoxon otherwise; for qualitative variables, patients were compared by parametric tests of $\mathrm{x} 2$ or nonparametric Fisher when the number is less than 5 .

\section{Results}

Between December 2015 and October 2017, 122 patients who underwent RARP by one experienced surgeon (SV) were included. As shown figure 1, 97 patients were included in the analysis. 
The mean age of the population was $62.8 \pm 5.5$ years. The ASA score was 2 for $62 \%$ of patients and only $1 \%$ had an ASA score of 3 . The most common comorbidity was high blood pressure (31\%). Demographics are described Table 1. The duration of the surgery was $2.5 \mathrm{~h} \pm 0.4$. Three intraoperative complications were reported: a severe bradycardia requiring atropine administration, a difficult intubation, and a minor subcutaneous emphysema that did not necessitate the interruption of the procedure or conversion. $54 \%$ of the patients were discharge from PACU after 5:00pm. Intraoperative data are detailed Table 2.

Postoperative data are described Table 3. Only 1 patient had a PADSS score $\geq 9$ at D0, seventy-two patients had a PADSS score $\geq 9$ at D1 and 25 patients at D2 or D3. While $74 \%$ of the patients had discharge criteria one day after surgery, $33 \%$ and $66 \%$ of them was effectively discharged on day 2 and day 3 , respectively. There were no 30-day readmissions.

First flatus appeared at D0 for only $4 \%$ of the patients and at D1 for $76 \%$. The vast majority of the patients (99\%) walked on D1. Nurses interventions more than usual monitoring were needed for $38 \%$ of the patients (the most frequent for pain, vagal types of adverse reaction or blood spot on bandages). These extra interventions led to a change in management compared with the usual protocol in only $16 \%$ of the patients. There were very few complications, only two renal failure rapidly resolved after IV rehydration. The most frequent complication was PONV (12\%).

When comparing patients with a PADSS score $\geq 9$ at D1 and patients with a PADSS score $\geq 9$ at D2 or D3, the preoperative data were comparable except for the BMI which was significantly higher in patients with PADSS score $\geq 9$ at D1 than patients with PADSS score $\geq 9$ at D2 or D3 $(26.1 \pm 3.1$ vs $24.1 \pm 2.6 ; p=0.0046)$. The 
intraoperative data were also comparable. Postoperatively, patients with a PADSS score $\geq 9$ at D1 experienced significantly less PONV than patients with a PADSS score $\geq 9$ at D2 or D3 (7\%vs $28 \%, p<0.01)$. Analgesic consumption was comparable. We also observed more nurses intervention in patients with PADSS score $\geq 9$ at D2 or $3(80 \%$ vs $24 \% ; p<0.0001)$, more medical call $(40 \%$ vs $10 \% ; p=0.0015)$, medical interventions $(32 \%$ vs $4 \% ; p=0.0007)$ and more deviations to standard protocol (32\% vs $11 \% ; p=0.0260)$.

\section{Discussion}

In this study, after a RARP only one patient achieved discharge criteria at D0. However, $74 \%$ of the patients had-discharge criteria at $\mathrm{D} 1$ and $26 \%$ at $\mathrm{D} 2$.

The International Association for Ambulatory Surgery (IAAS) does not define the types of surgery eligible for ambulatory (17). Improvements in surgical and anesthetic techniques allow outpatient surgery for many types of surgery. For example, in orthopedics, outpatient surgery was described for anterior cervical discectomy with a success rate of $90 \%$. Indeed, 27 out of the 30 patients studied were discharged the same day of surgery with a satisfaction rate of 9.6/10 (18). Several studies also reported cases of total hip arthroplasty in outpatients. For example, den Hartog et al. showed that 24 patients out of the 27 studied were discharged the same day of the surgery without complications or readmission, the other 3 patients were not discharged due to PONV and/or dizziness (19). A retrospective study compared 63,424 total hip arthroplasty surgeries with a hospital stay of 1 to 5 days with 420 outpatient total hip replacement surgeries and found no difference in terms of complications or readmission (20). In abdominal surgery, fast-track recovery program have long been developed and a recent article described 5 patients with a colectomy were discharged within 12 hours after the surgery (9). 
Regarding radical prostatectomy, length of stay after prostatectomy has rapidly evolved in the recent years: a systematic review reported 7.87 days for open surgery, 6.09 days for laparoscopic surgery and 3.85 days for RARP in 2014 (21). In 2016, a study comparing RARP to open surgery reported length of stay as short as 1.55 days and 3.27 days, respectively ${ }^{7}$. Previous publications have already evaluated the feasibility of outpatient RARP. In 2010, a first study performed RARP in 11 outpatients without complications and with an excellent satisfaction score (10). In 2016, a French case report described the case of a 57-year-old patient who was able to be discharged less than 12 hours after surgery (22). A prospective study including 30 highly selected patients reported that $87 \%$ of the patients were discharged the same day of the surgery without postoperative complications and with excellent patient satisfaction (23).

In our study, the main risk factor for delayed discharge was PONV. PONV is commonly reported after abdominal surgery. Some studies have shown a higher rate of PONV in laparoscopic surgery compared to open surgery (24)(25). More recently, one study had shown a higher rate of PONV in robotic surgery, with $33 \%$ of PONV after RARP versus $16 \%$ after laparoscopic prostatectomy, probably due to steeper head-down position and prolonged pneumoperitoneum (26). In our study, overall incidence of PONV was $12 \%$. Our protocol included PONV prophylaxis with intraoperative dexamethasone and postoperative ondansetron if needed. The lower incidence of PONV observed in our study was probably due to the prophylaxis. However, despite this protocol, up to $28 \%$ of the patient with delayed discharge reported PONV, suggesting intensification of the prophylaxis may be required.

In our experience, late PACU discharge after 5pm happened in 54\% of the patients. Late return from PACU preclude any chance of DO discharge and outpatient 
management in French institutions. Indeed, according to the French definition of outpatient surgery and because of the closing constraints of the outpatient surgery department, patients have to leave the hospital before 9:00 pm. However, the definition of outpatient surgery varies widely in the literature. In France, the code of public health defines outpatient surgery as a hospital stay of less than or equal to 12 hours not including a night in hospital (27). The IAAS defines true ambulatory surgery as a discharge during the time frame of one working day (6 to 8 hours) with no overnight stay and ambulatory surgery with extended recovery with a stay for one night postoperatively in a hospital facility (overall stay up to 23 hours) (28). However, previous studies on outpatient RARP did not specify whether it was true ambulatory or an ambulatory surgery with extended recovery, for example, Berger et al. compared outpatient and inpatient group after RARP but in the outpatient group, 87\% of the population were discharge the same day of surgery and the other stayed overnight with a mean length of stay of $14 \mathrm{~h}$ for the outpatient group (23). In our study, the PADSS score at D1 was not evaluated in the morning but rather in the afternoon. Therefore, it could be considered that most of our patients were likely to be ready for discharge within the 24 postoperative hours and were eligible to outpatient discharge with extended recovery.

In order to achieve outpatient RARP, we applied recommendations for enhanced recovery after surgery (ERAS). ERAS combines pre-, intra-, and postoperative measures adapted to the surgery to counteract or minimize the deleterious effects of surgery and/or anesthesia. ERAS was initially implemented in colorectal surgery and is now developed for various types of surgeries ${ }^{8}$. Guidelines for colorectal surgery combine among other things, preoperative information, a reduce preoperative fasting (6h for solids and $2 \mathrm{~h}$ for liquids) with administration of carbohydrate-rich isotonic 
fluids, intraoperative monitoring fluid administration based, hypothermia and PONV prevention and postoperative multimodal analgesia privileging non-opioid drugs, adequate thromboprophylaxis, enforced patient mobilization and early oral feeding. These guidelines could apply for RARP. However, specific guidelines remain to be publish. Opioid free anesthesia seems to demonstrate a decrease in postoperative pain and PONV ${ }^{29}$ which could also allow an earlier discharge of patients. In our study, preoperative counseling was probably missing and could improve earlier discharge when implemented.

Our study has some limitations. It is an observational and monocentric study. However, all patients received a pre-defined standard anesthesia protocol. All RARP were performed by a single and experienced surgeon. Discharge was only considered on PADSS score and PADSS score may have missed clinical or institutional limitation that preclude early discharge. Indeed, items like comfort, willingness and/or readiness to go home, fatigue, eating well, ileus, etc...could also be determinant for discharge.

In conclusion, discharge at DO after RARP was not feasible in our study. Discharge at D1 was however possible. It is a significant improvement over an effective discharge at D3. Reinforcing PONV prophylaxis and institution organization would probably allow earlier discharge and probably outpatient management. In this context, an opioid free anesthesia could be proposed and needs to be further studied. 


\section{References}

1. Fact Sheets by Cancer. http://globocan.iarc.fr/Pages/fact_sheets_cancer.aspx. Accessed February 15, 2018.

2. Center MM, Jemal $\mathrm{A}$, Lortet-Tieulent $\mathrm{J}$, et al. International Variation in Prostate Cancer Incidence and Mortality Rates. Eur Urol. 2012;61(6):1079-1092.

3. Yu H, Hevelone ND, Lipsitz SR, Kowalczyk KJ, Hu JC. Use, Costs and Comparative Effectiveness of Robotic Assisted, Laparoscopic and Open Urological Surgery. J Urol. 2012;187(4):1392-1399.

4. Tollefson MK, Frank I, Gettman MT. Robotic-assisted Radical Prostatectomy Decreases the Incidence and Morbidity of Surgical Site Infections. Urology. 2011;78(4):827-831.

5. C. Barchi L. Applications and Economics Aspects of Robotic Surgery. Int J Adv Robot Autom. 2016;1(2):1-6.

6. Ilic D, Evans SM, Allan CA, Jung JH, Murphy D, Frydenberg M. Laparoscopic and robot-assisted vs open radical prostatectomy for the treatment of localized prostate cancer: a Cochrane systematic review. BJUInt::n/a-n/a.

7. Yaxley JW, Coughlin GD, Chambers SK, et al. Robot-assisted laparoscopic prostatectomy versus open radical retropubic prostatectomy: early outcomes from a randomised controlled phase 3 study. The Lancet. 2016;388(10049):1057-1066.

8. Alfonsi $\mathrm{P}$, Slim K, Chauvin M, Mariani P, Faucheron JL, Fletcher D. French guidelines for enhanced recovery after elective colorectal surgery. J Visc Surg. 2014;151(1):65-79.

9. Gignoux B, Pasquer A, Vulliez A, Lanz T. Outpatient colectomy within an enhanced recovery program. J Visc Surg. 2015;152(1):11-15.

10. Martin AD, Nunez, RN, Andrews JR, Martin GL, Andrews PE, Castle EP. Outpatient Prostatectomy: Too Much Too Soon or Just What the Patient Ordered. Urology. 2010;75(2):421-424.

11. Antibioprophylaxie en chirurgie et médecine interventionnelle (patients adultes). Société Française d'Anesthésie et de Réanimation. http://sfar.org/antibioprophylaxie-en-chirurgie-et-medecine-interventionnellepatients-adultes-2017/. Published September 22, 2017. Accessed May 7, 2018.

12. Geerts WH, Bergqvist D, Pineo GF, et al. Prevention of Venous Thromboembolism: American College of Chest Physicians Evidence-Based Clinical Practice Guidelines (8th Edition). CHEST. 2008;133(6):381S-453S.

13. Chung F, Chan VW, Ong D. A post-anesthetic discharge scoring system for home readiness after ambulatory surgery. J Clin Anesth. 1995;7(6):500-506. 
14. Chung F. Recovery pattern and home-readiness after ambulatory surgery. Anesth Analg. 1995;80(5):896-902.

15. Clavien PA, Barkun J, de Oliveira ML, et al. The Clavien-Dindo Classification of Surgical Complications: Five-Year Experience. Ann Surg. 2009;250(2):187.

16. Khwaja A. KDIGO Clinical Practice Guidelines for Acute Kidney Injury. Nephron. 2012;120(4):c179-c184.

17. IAAS Recommendations. http://www.iaas-med.com/index.php/iaasrecommendations. Accessed June 20, 2018.

18. Gennari A, Mazas S, Coudert P, Gille O, Vital J-M. Outpatient anterior cervical discectomy: a french study and literature review. Orthop Traumato/ Surg Res.

19. den Hartog YM, Mathijssen NMC, Vehmeijer SBW. Total hip arthroplasty in an outpatient setting in 27 selected patients. Acta Orthop. 2015;86(6):667-670.

20. Nelson SJ, Webb ML, Lukasiewicz AM, Varthi AG, Samuel AM, Grauer JN. Is Outpatient Total Hip Arthroplasty Safe? J Arthroplasty. 2017;32(5):1439-1442.

21. Carlo FD, Celestino F, Verri C, Masedu F, Liberati E, Stasi SMD. Retropubic, Laparoscopic, and Robot-Assisted Radical Prostatectomy: Surgical, Oncological, and Functional Outcomes: A Systematic Review. Urol Int. 2014;93(4):373-383.

22. Leclers $F$, Dutheil V, Poupot $\mathrm{D}$, et al. La prostatectomie radicale robotique ambulatoire. Une chirurgie de T'avenir? À propos d'un cas. Prog En Urol. 2016;26(1):10-13.

23. Berger AK, Chopra S, Desai MM, Aron M, Gill IS. Outpatient Robotic Radical Prostatectomy: Matched-Pair Comparison with Inpatient Surgery. J Endourol. 2016;30(S1):S-52.

24. litomi T, Toriumi S, Kondo A, Akazawa T, Nakahara T. Incidence of nausea and vomiting after cholecystectomy performed via laparotomy or laparoscopy. Masui. 1995;44(12):1627-1631.

25. Watcha MF, White PF. Postoperative Nausea and Vomitinglts Etiology, Treatment, and Prevention. Anesthesiol J Am Soc Anesthesiol. 1992;77(1):162184.

26. Yonekura $\mathrm{H}$, Hirate $\mathrm{H}$, Sobue $\mathrm{K}$. Comparison of anesthetic management and outcomes of robot-assisted vs pure laparoscopic radical prostatectomy. J Clin Anesth. 2016;35:281-286.

27. Décret No 2012-969 Du 20 Août 2012 Modifiant Certaines Conditions Techniques de Fonctionnement Des Structures Alternatives à l'hospitalisation Article 2.; 2012.

28. Extended Recovery Facilities. http://www.iaas-med.com/index.php/iaasrecommendations/extended-recovery-facilities. Accessed June 20, 2018. 
29. Blaudszun G, Lysakowski C, Elia N, Tramèr MR. Effect of perioperative systemic a2 agonists on postoperative morphine consumption and pain intensity: systematic review and meta-analysis of randomized controlled trials. Anesthesiology. 2012;116(6):1312-1322. d

Legend of the figures:

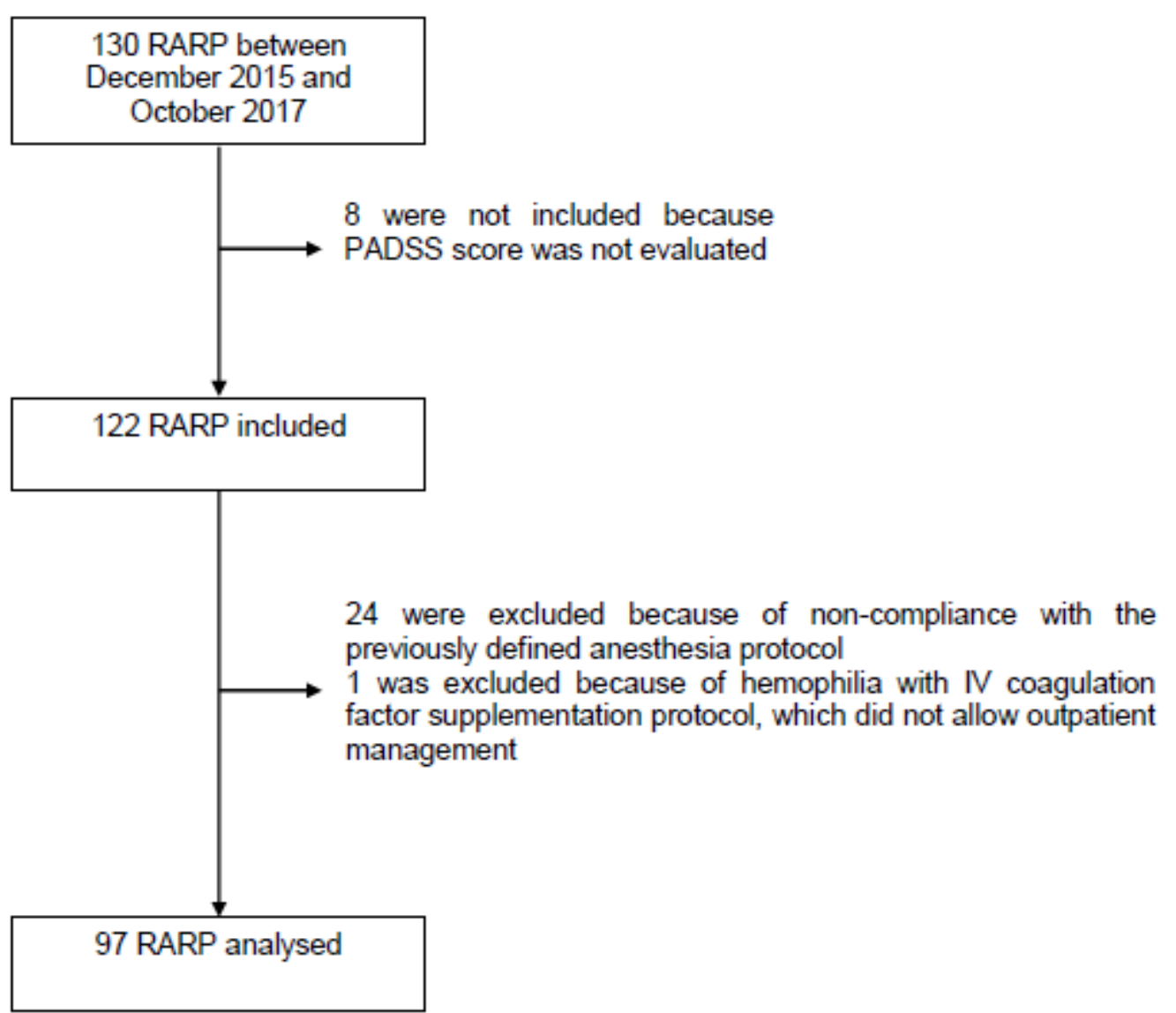

Figure 1: Flow chart 
Table 1: Preoperative data

Age

$62.8 \pm 5.5$

ASA score

1

2

3

$\mathrm{BMI}$

Diabetes

Kidney diseases

Respiratory diseases

Liver diseases

Vascular diseases

Coronary diseases

High blood pressure

Smoking

Blood disorders

Chronic Platelet antiaggregant

treatment

Chronic Anticoagulant treatment

Patient characteristics are described by mean \pm standard deviation (SD) for quantitative values and $n$ and percentage for qualitative values with $n$ global $=97$. BMl: Body mass index

$$
\begin{gathered}
36(37 \%) \\
60(62 \%) \\
1(1 \%)
\end{gathered}
$$$$
25.6 \pm 3.1
$$

$3(3 \%)$

$2(2 \%)$

$5(5 \%)$

$1(1 \%)$

$3(3 \%)$

$5(5 \%)$

$30(31 \%)$

$12(12 \%)$

$1(1 \%)$

$8(8 \%)$

$1(1 \%)$

\section{global = 97. BMI: Body mass index}


Table 2: Intraoperative data

Duration of surgery (h)

Bleeding more than $500 \mathrm{ml}$

Lymph node dissection

Nerve preservation

Bilateral

Unilateral

Intraoperative complications

Antibioprophylaxis

Duration PACU (h)

Discharge of PACU after 5:00pm

Morphine in PACU $(\mathrm{mg})$
$2.5 \pm 0.4$

$1(1 \%)$

$73(75 \%)$

$39(40 \%)$

$9(9 \%)$

$3(3 \%)$

$97(100 \%)$

$2.6 \pm 0.9$

$52(54 \%)$

$5.9 \pm 5.4$

Intraoperative data are described by mean \pm standard deviation (SD) for quantitative values and $n$ and percentage for qualitative values with $n$ global $=97$. h: hours, $\mathrm{mg}:$ milligram 
Table 3: Postoperative data

First liquid (h+ PACU discharge)

$1.5 \pm 1.7$

First food (h+ PACU discharge)

$5.6 \pm 3.6$

First flatus
D0
D1
D2

$4(4 \%)$

$74(76 \%)$

$19(20 \%)$

$40(41 \%)$

Chewing-gum protocol

$1(1 \%)$

D0

$96(99 \%)$

Perfusion catheter removal

D0

$1(1 \%)$

D1

$89(92 \%)$

D2

$7(7 \%)$

Postoperative protocol application

$62(64 \%)$

Oxycodone consumption (mg)

$1.5 \pm 3.6$

Ketoprofen consumption (mg)

$57.2 \pm 80.0$

Paracetamol consumption (g)

$4.4 \pm 2.0$

Nefopam consumption (mg)

$20.8 \pm 31.1$

Postoperative complications (Clavien-Dindo

classification)

Grade 1

$2(2 \%)$

Renal failure

PONV

$12(12 \%)$

Grade 2

0

Grade 3

0

Grade 4

0

Grade 5

Postoperative data are described by mean \pm standard deviation (SD) for quantitative values and $n$ and percentage for qualitative values with $n$ global $=97 . h+$ PACU discharge is number of hours after PACU discharge; mg: milligram, $g$ : gram 